\title{
Experimental studies on coherent synchrotron radiation at an emittance exchange beam line
}

\author{
J. C. T. Thangaraj, ${ }^{*}$ R. Thurman-Keup, J. Ruan, A. S. Johnson, A. H. Lumpkin, and J. Santucci \\ Fermi National Accelerator Laboratory, Batavia, Illinois 60510, USA
}

(Received 8 March 2012; published 19 November 2012)

\begin{abstract}
One of the goals of the Fermilab A0 photoinjector is to investigate experimentally the transverse to longitudinal emittance exchange (EEX) principle. Coherent synchrotron radiation in the emittance exchange line could limit the performance of the emittance exchanger at short bunch lengths. In this paper, we present experimental and simulation studies of the coherent synchrotron radiation (CSR) in the emittance exchange line at the A0 photoinjector. We report on time-resolved CSR studies using a skewquadrupole technique. We also demonstrate the advantages of running the EEX with an energy-chirped beam.
\end{abstract}

DOI: 10.1103/PhysRevSTAB.15.110702

PACS numbers: 41.60.Ap, 41.60.Cr, 41.75.Ht, 29.27.Bd

\section{INTRODUCTION}

The ability to manipulate the $6 \mathrm{D}$ phase space of the electron beam is important for both modern and future accelerators. One such phase-space manipulation scheme involves exchanging the longitudinal emittance with the transverse emittance of the beam. At the Fermilab A0 photoinjector, a proof-of-principle experiment of an emittance exchange (EEX) scheme has been demonstrated recently [1]. The emittance exchange line consists of a $\mathrm{TM}_{110}$ cavity sandwiched by doglegs, a variation of the original scheme proposed by Cornacchia and Emma [2], where the cavity was in the middle of a chicane. Neither scheme takes into account collective effects such as space charge, coherent synchrotron radiation, and wakefields.

Our goal in this work is to investigate coherent synchrotron radiation effects (CSR) in the emittance exchange line at the Fermilab A0 photoinjector. The A0 photoinjector has a history of experiments in subpicosecond pulse train generation, flat-beam generation, electro-optic sampling experiments, and photoinjector dynamics among others [3-6]. In this paper we begin with the measurement of CSR power as a function of charge and bunch length, followed by a report on the measurement of the angular distribution and polarization of the CSR. We then show how CSR was used to measure the bunch length of the electron beam and compare those results with numerical simulations. Next we show results from time-resolved studies of CSR using a skew quadrupole. Finally, we conclude with measurements from the emittance exchanger, where a peak current increase by a factor of 2 was obtained with an energy-chirped beam.

\footnotetext{
*jtobin@fnal.gov
}

Published by the American Physical Society under the terms of the Creative Commons Attribution 3.0 License. Further distribution of this work must maintain attribution to the author(s) and the published article's title, journal citation, and DOI.

\section{THEORY}

Synchrotron radiation is the electromagnetic radiation emitted by a radially accelerated charged particle. CSR refers to synchrotron radiation with a wavelength that is longer than the bunch length and thus emitted coherently throughout the bunch [7]. The steady-state CSR power loss based on a free-space model can be expressed as [8]: $P=$ $\left(N^{2} x e^{2}\right) /\left(\epsilon_{0} R^{2 / 3} \sigma_{z}^{4 / 3}\right)$ Watts, where $N$ is the number of particles, $x$ is $0.0279, R$ is the bending radius in $m, \sigma_{z}$ is the rms bunch length in $m$, and $e$ is the charge of electron. Therefore, the shorter the bunch at the dipole, the larger the power loss due to CSR. This formula assumes: (i) that the length of the bend $L_{b}$ is longer than the overtaking length $\mathrm{E}_{\mathrm{o}}=2\left(3 \sigma_{\mathrm{z}} \mathrm{R}^{2}\right)^{1 / 3}$; (ii) the vacuum pipe radius is larger than the suppression parameter for wave propagation $r_{b}=2\left(9 \sigma_{z}^{2} R\right)^{1 / 3}$; and (iii) that transient and transverse effects are ignored. Transverse effects can be ignored if $\sigma_{x} /\left[\left(\sigma_{z}^{2} R\right)^{1 / 3}\right] \ll 1$, where $\sigma_{x}$ is the rms transverse beam size of the bunch [8].

The total emitted power, $P$, can be shown to be equal to $P=N P_{\text {inc }}[1+N g(\lambda)]$, where $P_{\text {inc }}$ is the single-particle classical synchrotron radiation power, $N$ is the number of particles, and $g(\lambda)$ is the form factor proportional to the Fourier transform of the longitudinal profile of the bunch. If the condition for coherency is satisfied, i.e. $N g(\lambda) \gg 1$, the single-particle radiation intensity is multiplied by the bunch form factor and $N^{2}$ to yield CSR. For a Gaussian bunch with rms bunch length $\sigma_{z}$, the form factor $g(\lambda)=$ $\exp \left[-\left(\frac{2 \pi \sigma_{z}}{\lambda}\right)^{2}\right]$, indicating that bunches will emit CSR at wavelengths longer than $2 \pi \sigma_{z}$. A complete discussion on the formation length and transverse effects of CSR are given in [9].

In addition to the average energy loss due to CSR, the radiation from the tail of the bunch can catch up with the head of the bunch and interact leading to an energy spread within the bunch. The energy spread is then converted to a 


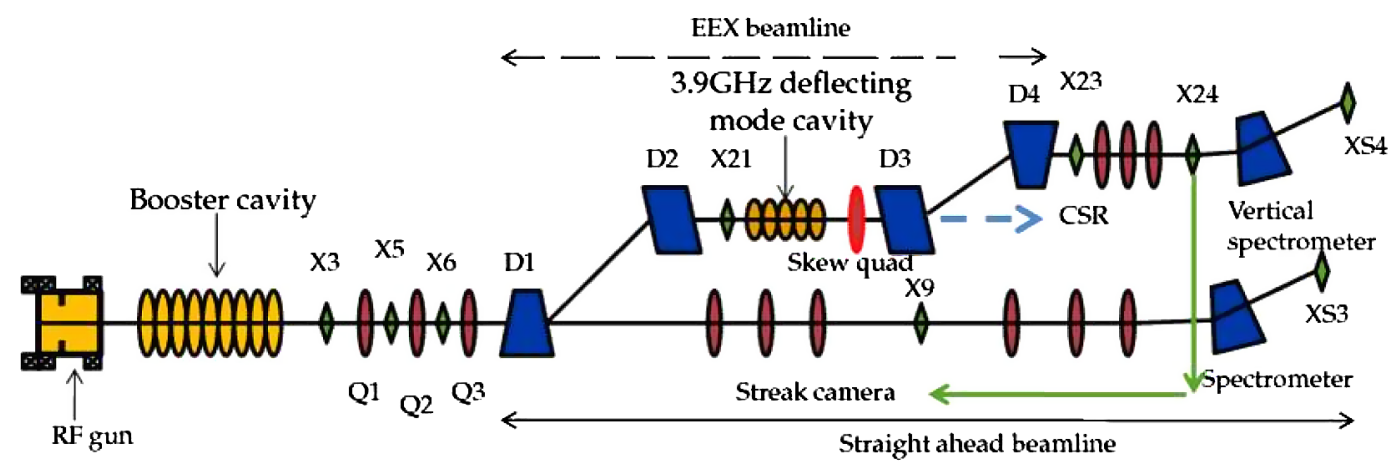

FIG. 1. Experimental setup of the A0 photoinjector facility. Electrons generated by the rf gun are accelerated through the booster cavity to $14 \mathrm{MeV}$. Q1, Q2, and Q3 focus the beam before it enters the EEX beam line. D1, D2, D3, and D4 are the dipole magnets. The skew quadrupole located before D3 is used to study time-resolved effects. X\# refers to beam imaging stations.

projected emittance growth when the bunch exits a dispersive section such as a bend. Some recent experimental studies on CSR in a chicane are in Refs. [10-12].

\section{EXPERIMENTAL SETUP}

The A0 photoinjector facility (A0PI) shown in Fig. 1 consists of an L-band rf gun followed by a superconducting 9-cell booster cavity, which accelerates the electron beam up to $16 \mathrm{MeV}$. After acceleration, the beam is steered and focused using the dipoles and the quadrupoles (Q1 Q2 Q3). The beam can either continue straight to XS3 or be steered into the dogleg. In our experiments, the beam is sent through the doglegs to the spectrometer (XS4). Located between the doglegs is the liquid-nitrogen-cooled, 5-cell 3.9 $\mathrm{GHz} \mathrm{TM}_{110}$ deflecting mode cavity, which was switched on/off during our study. The nominal parameter set used in A0 simulation is shown in Table I. The precise parameter set for the emittance exchange experiment is given in [1].

When the bunch passes through the dogleg, CSR is expected to be more pronounced at dipole D3 for our experimental parameters (see Appendix A). We installed optics to collect the radiation coming out of the port at dipole D3. The light is collimated using an off-axis parabolic mirror onto a plane mirror. The reflected light is then

TABLE I. Nominal A0 beam parameters.

\begin{tabular}{lcc}
\hline \hline Parameter & Value & Units \\
\hline Beam energy & 14.3 & $\mathrm{MeV}$ \\
Charge & 250 & $\mathrm{pC}$ \\
Transverse emittance (normalized) & 4 & $\mu \mathrm{m}$ \\
Longitudinal emittance (normalized) & 18 & $\mu \mathrm{m}$ \\
Dipole bending radius & 0.9 & $\mathrm{~m}$ \\
Dipole bending angle & 22.5 & degrees \\
Chamber height & 18 & $\mathrm{~mm}$ \\
Initial bunch length & 3 & $\mathrm{ps}$ \\
Bunch length at dipole D3 (rf-chirp dependent) & $0.5-0.8$ & $\mathrm{ps}$ \\
\hline \hline
\end{tabular}

directed either to a single $\mathrm{LiTaO}_{3}$ crystal pyrodetector for power measurement or to a Martin-Puplett interferometer to measure bunch length [13]. The layout of the collecting optics is shown in Fig. 2.

\section{A. CSR power measurements}

At the A0 photoinjector, the CSR originates from the dipole bending magnets in the dogleg sections. The intensity of CSR scales as $N^{2}$, where $N$ is the number of particles in the bunch. We measured the intensity of the radiation for various bunch charges and at different $\mathrm{rf}$ phases. For a fixed charge, we found that the detector power is maximum at the minimum bunch length at D3. Moreover, the detected power also varies quadratically with charge as expected for coherent radiation, and this is shown in the Fig. 3 inset. In order to prevent the pyrodetector from saturation at higher charge, we chose 10 bunches per pulse train.

As seen in Fig. 3, the CSR power peaks at $41^{\circ}$ off crest, and this feature can be explained as follows. The final

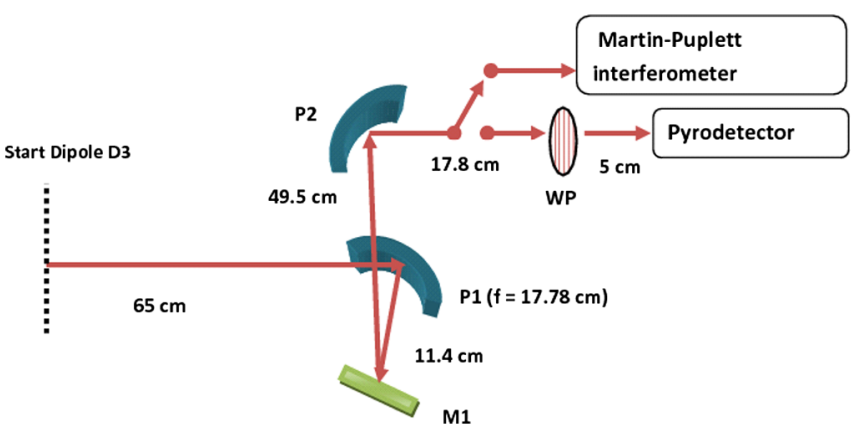

FIG. 2. Schematic of the synchrotron light collection optics from dipole D3. CSR is first collected by an off-axis parabolic mirror ( $\mathrm{P} 1$ with focal length $=17.78 \mathrm{~cm}$ ) and focused on a mirror (M1) which reflects the light to another parabolic mirror (P2). P2 focuses the light onto a pyrodetector or a Martin-Puplett interferometer. The wire-grid polarizer (WP) was installed $5 \mathrm{~cm}$ before the pyrodetector. 


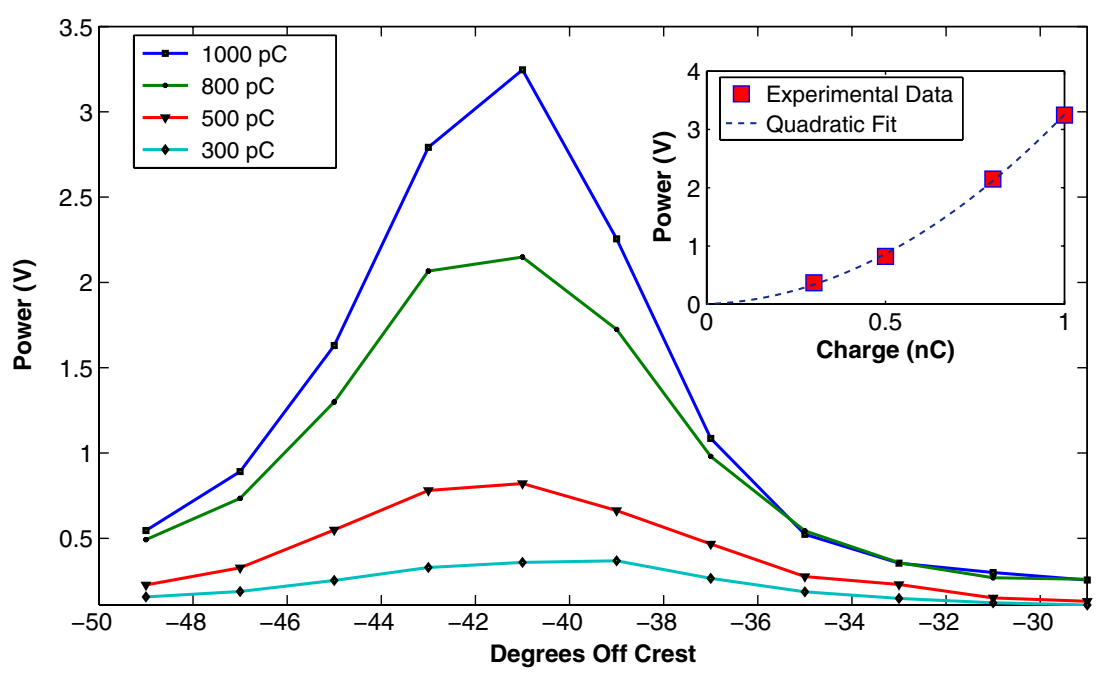

FIG. 3. CSR Power measurement at D3 as a function of 9-cell booster cavity phase. Maximum power is observed at $41^{\circ}$ off crest. The inset shows the fit of the CSR power as a function of charge.

bunch length $\sigma_{z f}$ of the beam after going through a bend system is $\sigma_{z f}=\sqrt{\left(1+\kappa R_{56}\right)^{2} \sigma_{z i}^{2}+\left(R_{56} \sigma_{\delta_{i}}\right)^{2}}$, where $\sigma_{z i}$ is the initial uncorrelated bunch length, $\sigma_{\delta_{i}}$ is the initial energy spread, $R_{56}$ is the longitudinal dispersion, and $\kappa$ is the linear chirp [14]. To minimize the bunch length, we must minimize the incoming energy spread and/or set the linear chirp of the rf 9-cell booster cavity to $\kappa=-\frac{1}{R_{56}}$. For the bending system (first dogleg alone in our case), $R_{56}=$ $0.12 \mathrm{~m}$, so the chirp should be $\kappa=-8.33$. For this chirp, the rf phase corresponds to $\phi=23^{\circ}$. Normally, to minimize the energy spread introduced by the electron gun, the rf phase is set to $\sim \phi_{0}=23^{\circ}(\kappa=-8.33)$. At this value of the rf phase, the beam has no chirp. In order to compress, we introduce an rf chirp on top of the regular offset phase. In other words, we should operate the 9-cell phase at a total chirp, $\kappa=-16.6$. This value of chirp is obtained for an $\mathrm{rf}$ phase off-crest value of $\phi=40$, which agrees well with the observed values.

\section{B. Polarization and angular distribution of CSR}

Since the bend plane is horizontal in the A0 doglegs, the synchrotron radiation is expected to be predominantly horizontally polarized. A wire-grid polarizer, which could be remotely rotated, was installed in front of the pyrodetector. The grids consist of $15 \mu \mathrm{m}$ diameter tungsten wires spaced by $45 \mu \mathrm{m}$. The intensity of the CSR was measured as a function of the polarizer angle and is shown in Fig. 4 along with a fit function. As expected, the light is mostly horizontally polarized. Assuming an electric field $\vec{E}_{x}$ and $\vec{E}_{y}$ for the horizontal and the vertical polarization components, incident at an angle of $\theta$ at the polarizer, the electric field after the polarizer is given by $\vec{E}_{t}=$ $\vec{E}_{x} \cos \theta+j \vec{E}_{y} \sin \theta$. The intensity at the detector is
$\left|E_{t}\right|^{2}=\vec{E}_{t} \cdot \vec{E}_{t}^{*}=E_{x}^{2} \cos ^{2} \theta+E_{y}^{2} \sin ^{2} \theta$. In order to fit the detector data, we model the intensity, $I=A^{2} \cos (X)+$ $B^{2} \sin (X)$, where we introduce $X=W \theta+\phi$ to add a weight $(W)$ to the angle of polarization $(\theta)$ and an offset $(\phi)$ to the polarizer angle.

To map the angular distribution of the light, the detector was mounted on an $X-Y$ translation stage and scanned over an area of $55 \mathrm{~mm} \times 60 \mathrm{~mm}$ with a 5-mm step size in each direction. The measurement was done for both horizontal and vertical polarizations, as shown in Fig. 5. The measured ratio between the horizontal and the vertical polarization components was 4.6. Theoretically, the horizontal polarization carries 7 times more power than the vertical

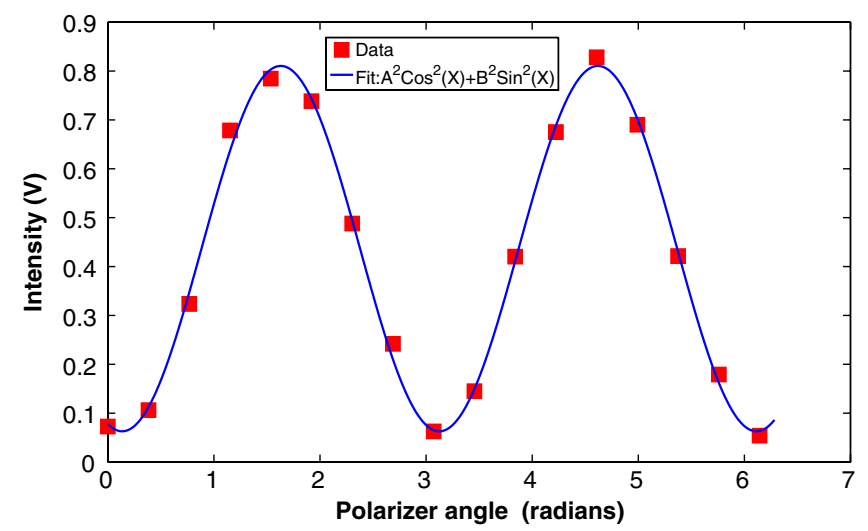

FIG. 4. The measured CSR intensity as a function of polarizer angle. While the sinusoidal behavior indicates a horizontally polarized CSR light, the nonzero baseline indicates the vertical polarization component of the CSR. The fit function is given by $A^{2} \cos ^{2}(X)+B^{2} \sin ^{2}(X)$, where $X=W \theta+\phi$ and $W, \phi, A$, and $B$ are arbitrary constants and $\theta$ is the polarizer angle in radians. From the fit we obtain $W=1.0510$ and $\phi=-0.1429$ radians. 

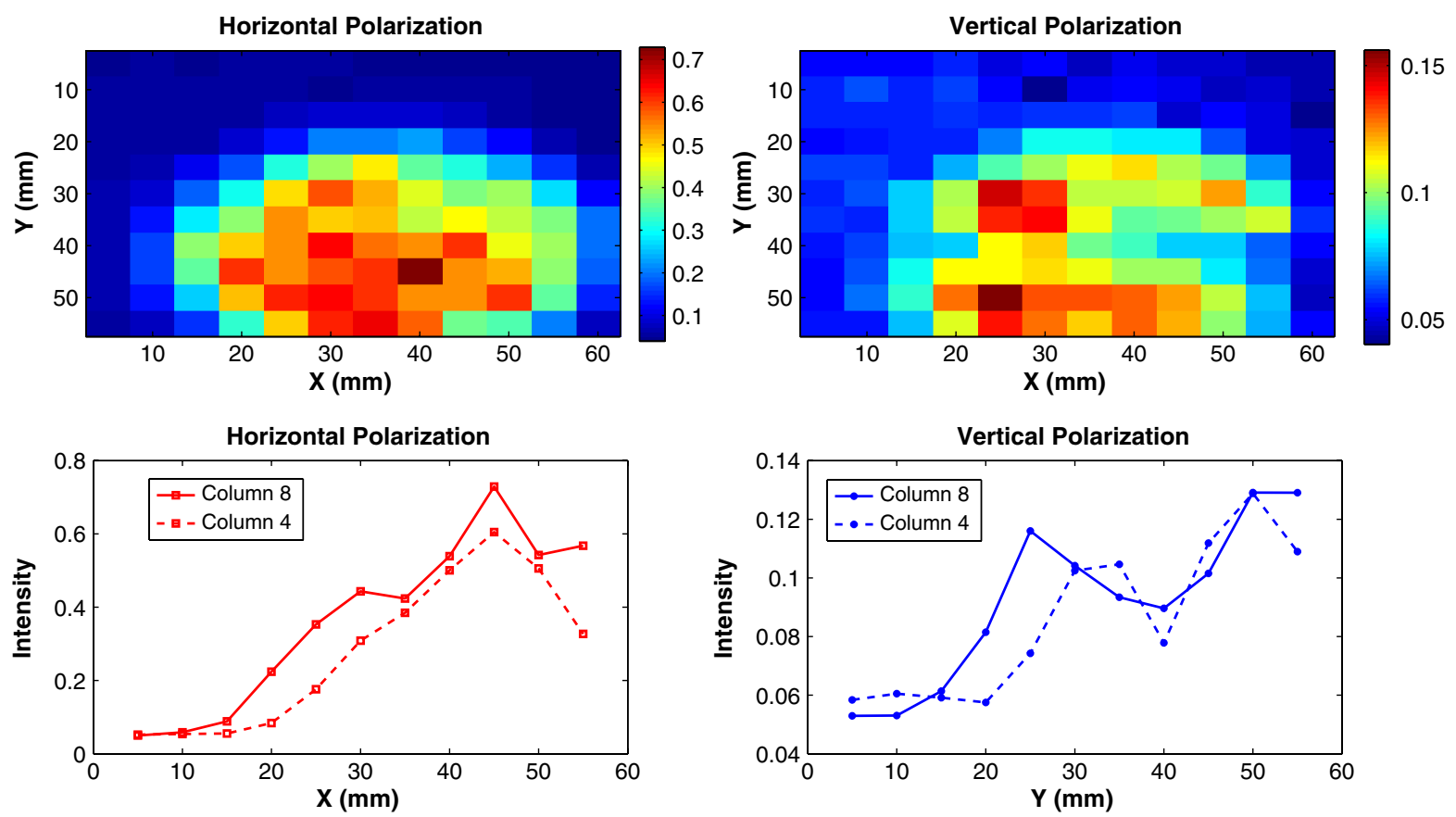

FIG. 5. Top: Measured angular distribution of the CSR radiation with horizontal polarization (left) and with vertical polarization (right). Bottom: Two slice projections from the spatial distribution showing the angular distribution of the coherent synchrotron radiation. The horizontal polarization (left) shows a maximum at the center-a single lobe, while the vertical polarization (right) shows a dip in the center-a double-lobed feature. Note: the scales are different by a factor of $\sim 5$ indicating higher intensity of the horizontally polarized synchrotron radiation.

[15]. The difference can be explained as follows: The bunch length $\left(\sigma_{z}\right)$ at dipole D3 is around $0.15 \mathrm{~mm}$ $(0.5 \mathrm{ps})$ corresponding to a wave number of $k_{s}=\frac{1}{\sigma_{z}}=$ $6667 \mathrm{~m}^{-1}$. The critical frequency of the dipole D3 is $k_{c}=\frac{3 \gamma^{3}}{2 R}=40229 \mathrm{~m}^{-1}$, where $\gamma$ is the relativistic factor and $R$ is the dipole bending radius. When $k_{s} \ll k_{c}$ and assuming no microbunching, CSR is expected only for frequencies $\leq k_{s}$. At such low frequencies, the horizontal

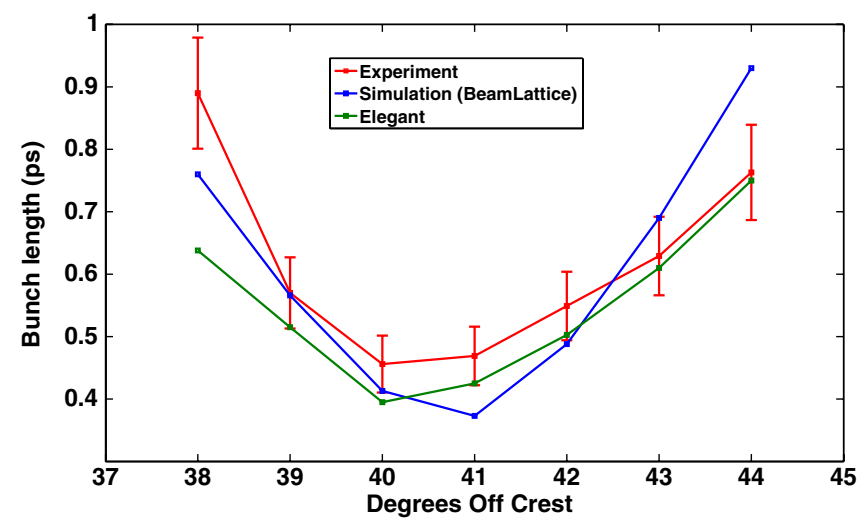

FIG. 6. Root-mean square bunch-length comparison for an ELEGANT simulation including CSR, a single-particle-matrix simulation without CSR, and the measurements. The charge was $250 \mathrm{pC}$. components are not as strong compared to vertical components as shown in [16], which agrees with the measurements. The difference may also be due to several other factors: in the experiment we are limited by the finite size of the detector, finite geometry of the beam pipe, and the diffraction limitation of the relay optics [17,18]. Also, shown in Fig. 5 is a slice of the spatial distribution showing the angular distribution of the CSR. The dip in the vertical angular distribution of the classical synchrotron radiation is observed.

\section{Bunch-length measurements}

Coherent synchrotron radiation carries the bunch-profile information of the electron beam in its bunch form factor. In order to extract the bunch length, the CSR was directed into a Martin-Puplett interferometer. A Martin-Puplett interferometer is a polarizing-type interferometer. The incoming light is first sent through a horizontal polarizer. Then, the horizontally polarized light wave is split using a beam splitter and directed to their respective arms of the interferometers. Once split, the light waves travel toward the roof mirror, which changes the polarization of the waves, and reflects them back to the beam splitter, where they are recombined. The recombined waves are again split and sent to pyrodetectors. The pyrodetector signals are digitized and read by a MATLAB routine to construct an autocorrelation trace to calculate the bunch length [13]. 

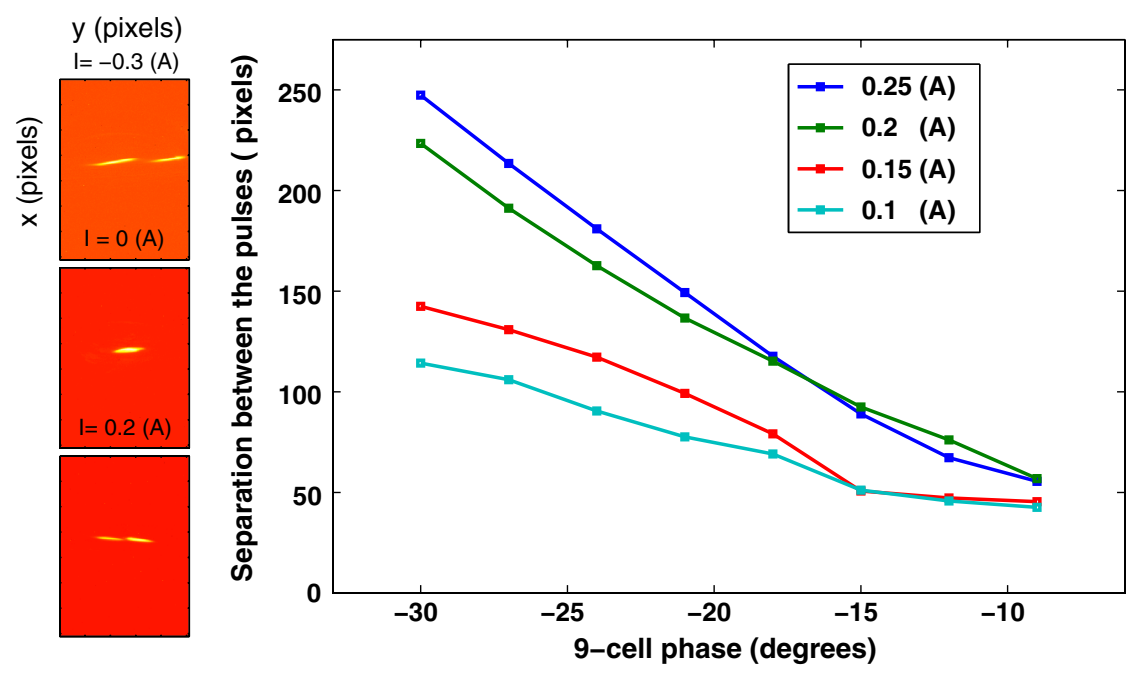

FIG. 7. The figures on the left show the transverse images of the twin pulses observed at X24 versus the skew-quadrupole current in Amps. When the skew quadrupole is turned on, the separation in pulses is observed. Each image is 520 pixels $\times 710$ pixels. The calibration factor is 32.8 microns/pixel. On the right, the separation of the pulses in pixel units are given as a function of rf chirp for every skew-quadrupole strength.

The results of the measurements and a comparison with the single-particle simulation using both BEAMLATTICE [19]a program based on a linear matrix model-and ELEGANT [20] are shown in Fig. 6. The uncertainty in the measurement is primarily due to the choice of the fitting function (low-frequency Taylor asymptote, low-frequency Gaussian asymptote) used to best model the low-frequency cutoff of the detector $[21,22]$. Simulation agrees with the experimental value for shorter bunch length while at longer bunch lengths low-frequency diffraction effects dominate, leading to the discrepancy. Also, the predicted rf phase at which the bunch length is minimum shows good agreement with the experimental value. The discrepancy between ELEGANT and BEAMLATTICE might be due to the absence of a second-order effects and CSR in BEAMLATTICE.

\section{Skew quadrupole}

Motivated by the work at SLAC that used a skew quadrupole to study CSR effects in a bunch compressor [23], a skew quadrupole was installed before the dipole D3. At the skew quadrupole, the particle gets a $y$ kick proportional to its $x$ position, $y^{\prime}=\frac{x}{F}$, where $F$ is the focal length of the skew quadrupole. This kick is converted to a $y$-position change at the screen (X24) downstream of the exchanger: $y_{\text {screen }}=R_{34} y_{\text {quad }}^{\prime}$. The subscript quad indicates the skew quadrupole. In terms of rms quantities, as long as the $\beta_{y}$ at the screen is small, the $y$ beam size at the screen is $\sigma_{y \text { screen }}=\frac{\sigma_{x \text { quad }}}{K}$, where $K$ is a constant and $\sigma_{x \text { quad }}=$ $\sqrt{x_{\beta \text { quad }}+\left(\eta_{x} \sigma_{\delta}\right)^{2}}$, where $\eta_{x}$ is the dispersion, $x_{\beta \text { quad }}$ is the beta function at the quad, and $\sigma_{\delta}$ is the rms energy spread. We consider two cases: with and without the 5-cell cavity powered.

\section{With 5-cell off}

If the beam size at the skew quadrupole is dominated by the dispersion term (as in a bunch compressor) and assuming linear chirp $h\left(\sigma_{\delta}=h \sigma_{z_{\text {in }}}\right)$, we can write $\sigma_{y \text { screen }}=$ $\frac{\eta_{x} h \sigma_{z_{\text {in }}}}{K}$. After canceling dispersion, the $\sigma_{y \text { screen }}$ is proportional to incoming bunch length. In our setup, the dispersion is canceled by using the last three quads after the X23 screen station.

\section{With 5-cell on}

If the 5-cell is on and the rf chirp is zero (at minimum energy spread), then we can write $y_{\text {screen }}=\sqrt{x_{\beta \text { quad }}} / K \propto$ $\sqrt{x_{0}^{2}+L^{2} x_{0}^{\prime 2}} / K$, where $L$ is the length of the dogleg drift,

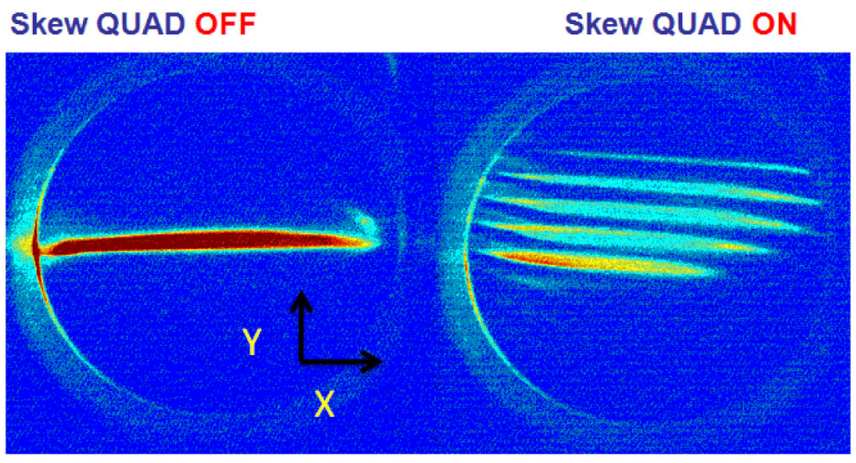

FIG. 8. Measured transverse beam images at X23 with the slits in at X3 and the 5-cell turned on. The skew-quadrupole projects the energy spectrum on the screen when the chirp is zero due to the leakage of the horizontal dispersion to the vertical plane. The observed separation of the slits in the $y$ direction is in good agreement with the simulation shown in Fig. 9. 

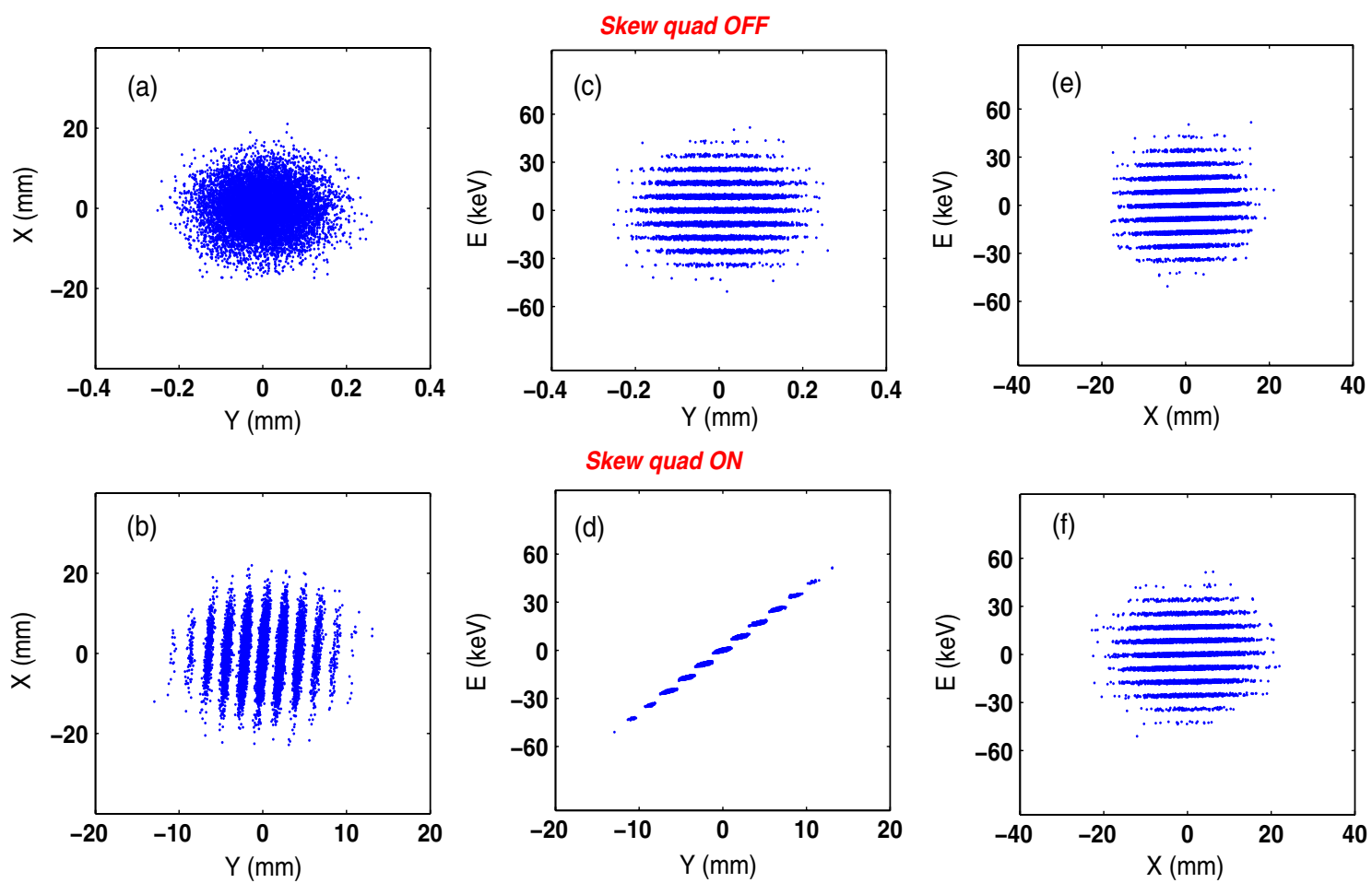

FIG. 9. BEAMLATTICE results showing the effect of the skew quadrupole at X23 with slits in at X3 and 5-cell on. Parts (a) and (b) show $X$ vs $Y$ transverse dimensions with the skew quadrupole off and on. Parts (c) and (d) show energy vs $Y$ with the skew quadrupole off and on. Turning on the skew quadrupole couples dispersion in the vertical plane introducing a strong correlation between $E$ and $Y$ as shown in part (d) which is mapped on the screen thus acting as a vertical spectrometer [part (b)]. The $X$ vs $E$ plane shown in parts (e) and (f) is unaffected by turning on the skew quadrupole. The rms transverse beam sizes for the case with skew quad off at X23 was $\sigma_{x}=2.3 \mathrm{~mm}$ and $\sigma_{y}=0.26 \mathrm{~mm}$ and for the case with skew quad on was $\sigma_{x}=2.7 \mathrm{~mm}$ and $\sigma_{y}=1.7 \mathrm{~mm}$.

because the beam size at the skew quadrupole is now dominated by $\beta_{x}$. From EEX transport equations [1], we can write $\delta_{\text {out }}=\frac{-x_{0}}{\eta}-\frac{L x_{0}^{\prime}}{\eta}$, where $x_{0}$ and $x_{0}^{\prime}$ are the initial transverse $x$ position and the angle of the beam before the EEX. Also, for a single particle going through a dogleg (with no chirp), $x_{\text {quad }}=x_{0}+L x_{0}^{\prime}$. Hence, we can write $x_{\text {quad }}=\delta_{\text {out }} \eta$. In summary, when the chirp is zero and with the 5-cell on, the $y$ beam size maps the outgoing energy profile: $y_{\text {screen }} \propto \frac{\delta_{\text {out }} \eta}{\kappa_{0}}$, where $\kappa_{0}$ is a constant of proportionality. Thus, the skew quadrupole acts like a vertical spectrometer. In this analysis we have made a thin-lens approximation for the 5-cell. Also, we have made an assumption that the time and energy are linearly correlated at the accelerating cavity, which is not exactly true if the operating phase is close to the crest. In our case, since we are far off crest, this is not a bad approximation. Spacecharge effects are also ignored because the space-charge wake strength for our parameters is an order of magnitude less than the CSR wake [9].

\section{E. 5-cell off: Twin pulse transport}

To begin our study, we used two electron pulses separated in time $(\sim 11 \mathrm{ps}(3.3 \mathrm{~mm}))$ and then turned on the skew quadrupole to see whether the time separation

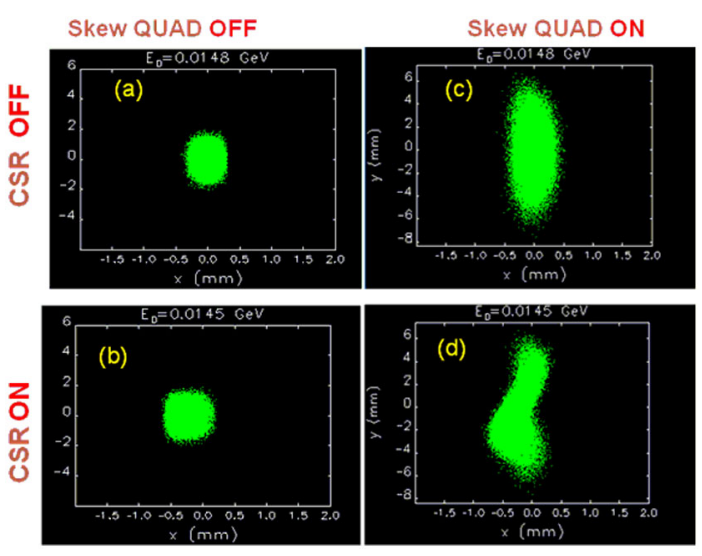

FIG. 10. ELEGANT simulation showing the effect of CSR and skew quadrupole at X24 with 5-cell off for $1 \mathrm{nC}$ charge bunch. Part (a) shows both CSR and the skew quadrupole off. When there is no CSR, the skew quadrupole acts like a vertical deflector as shown in part (c). Because of CSR, the head particles gain energy leading to a transverse offset as shown in part (d). The rms size at X24 is $\sigma_{x}=80 \mu \mathrm{m}$ and $\sigma_{y}=1.52 \mathrm{~mm}$. Part (b) shows the case with the skew quadrupole off and CSR on. 


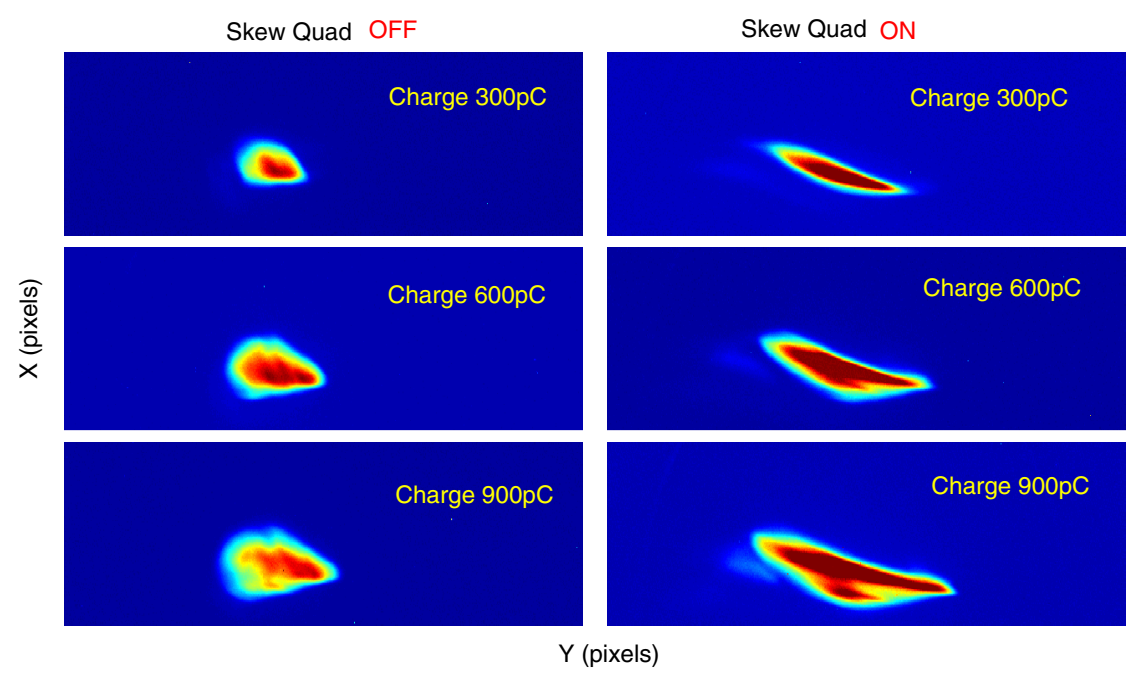

FIG. 11. Measured transverse beam profile at X24 as a function of skew quadrupole off (left column) and skew quadrupole on (right column) for various charges. The CSR bulge effect is prominent at higher charges. The image is rotated by $90^{\circ}$ compared to Fig. 10 . When the skew quadrupole is turned on, the $y$ axis on the right is proportional to the incoming bunch length and the $x$ axis proportional to the energy. Each image is 600 pixels $\times 150$ pixels with each pixel measuring 32.8 microns.

between the pulses could be revealed on the screen. The electron pulses separated in time experience different accelerating fields in the 9-cell and end up with different energies, but this does not show an $x$ position change at $\mathrm{X} 24$ because the dispersion is canceled using the quads before X24. By turning on the skew quadrupole, dispersion couples to the $y$ plane-thereby acting like a vertical deflector as shown in Fig. 7. As the skew quadrupole changes sign, the pulses flip their positions showing a direct correlation between the skew-quadrupole strength and time resolution at a fixed rf chirp. Also, we find that the larger the chirp, the more sensitive the time resolution is for a fixed skew current. Thus, the skew quadrupole can be used to time resolve the $x$ beam size at X24.

\section{F. 5-cell turned on: Slit-based measurements}

In order to study the effect of the skew quadrupole on the emittance exchange line, we switched on the 5-cell with the vertical slits inserted at X3. When the slits are inserted, the transverse modulation is transferred to longitudinal phase space leading to an energy and time modulation [24]. Hence at X23, the beam image has no modulation. But, when the skew quadrupole was turned on, the modulation effects are visible in the $y$ direction, as shown in Fig. 8 and can be explained as follows: when the chirp is zero (at minimum energy spread), the $x$ beam size at the skew quadrupole is dominated by $\beta_{x}$ and hence the skewquadrupole projects the $x$ modulation in $y$ at the screen. Any chirp then shows itself as the slit orientation at X23. However, the $x$ modulation at the skew quadrupole is proportional to the energy modulation at the output of the 5-cell. Hence, the skew quadrupole can be used to measure the energy spectrum of the beam exiting the
EEX. This is consistent with the simulation results from BEAMLATTICE as shown in Fig. 9.

\section{G. Time-resolved imaging of CSR effects}

We used ELEGANT to study the time-resolved effects of $\mathrm{CSR}$ at $\mathrm{X} 24$ by powering the skew quadrupole. We assumed a bunch charge $Q=1 \mathrm{nC}$, bunch length $\sigma_{z}$ of 3 ps. We also assumed emittances as $\epsilon_{x, y}=4 \mu \mathrm{m}$. After optimizing the beta function using ELEGANT for single-particle optics, we turned on CSR effects in ELEGANT by introducing CSRCSBEND and CSRDRIFT elements. The results are shown in Fig. 10. The signature of the CSR wake potential is clearly visible in the simulations. When CSR was turned on in the simulation, the particles near the head of the bunch distribution [see bottom of (d) in Fig. 10] are

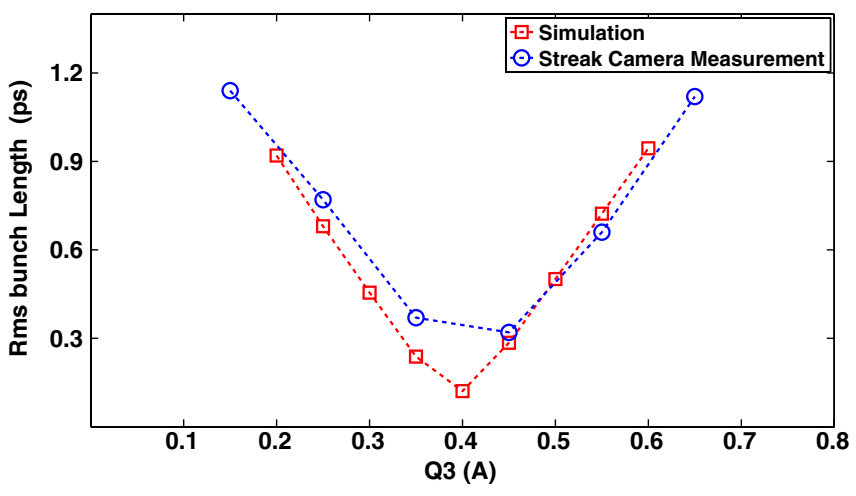

FIG. 12. Comparison of bunch lengths of the electron beam measured at X24 using a streak camera as a function of quadrupole (Q3) current versus those of simulation using BEAMLATTICE. The chirp was set to -40 degrees off crest. 
shifted horizontally by $0.35 \mathrm{~mm}$. We note here that the CSR model used in ELEGANT does not take into account transverse effects due to CSR which is important in our experiment. From [8], 3D effects can only be ignored if $\sigma_{x} /\left[\left(\sigma_{z}^{2} R\right)^{1 / 3}\right] \ll 1$, which is violated for our parameters $\left(\sigma_{x}=4.2 \mathrm{~mm}\right.$ and $\left.\sigma_{z}=0.8 \mathrm{ps}\right)$. Also, simulations have ignored collective effects like transverse space charge and wakefields.

The measured image profiles at X24 for various charges with and without the skew quadrupole on are shown in Fig. 11. As the charge increases from 300 to $900 \mathrm{pC}$, the CSR "bulge" effect is seen more pronounced on the screen as shown in Fig. 11. The shift in the $x$ position of the CSRshifted particles is compared with the rest of the bunch. For the $600 \mathrm{pC}$ case, the maximum offset is $0.49 \mathrm{~mm}$ while for the $900 \mathrm{pC}$ case, the maximum offset observed is $0.72 \mathrm{~mm}$. This is consistent with the CSR power measurement in Fig. 3, where increase in charge shows an increase in CSR power loss indicating that the CSR effect is more pronounced at higher charges.

\section{EFFECT OF CSR ON EMITTANCE EXCHANGER}

In all our experiments mentioned above to measure CSR, we had to impart an energy chirp on the beam using the 9-cell accelerating cavity. We show in this section that running the full emittance exchanger (which is typically run with no energy chirp) with an energy-chirped beam has advantages albeit with CSR energy loss.
In the emittance exchange beam line, if we assume a thick lens for the cavity, the transfer matrix is altered with coupling from incoming $z$ and $\delta$ [1]. So, the outgoing particle $z_{2}=\kappa \xi x_{1}+\left[\frac{-1}{\kappa}+\left(\frac{L_{c}}{4}+L\right) \xi\right] \kappa x_{1}^{\prime}+$ $\frac{L_{c} \kappa^{2} \xi}{4}\left(z_{1}+\xi \delta_{1}\right)$ where $\xi$ is the longitudinal dispersion, $x_{1}, x_{1}^{\prime}, z, \delta$ are the incoming $x$ position, $x$ angle, longitudinal position, energy spread of the beam and $L_{c}, L, \kappa$ are the length of the cavity, the length of the dogleg, and the strength of the cavity, respectively. If we set the chirp $=$ $\frac{\delta_{1}}{z_{1}}=\frac{-1}{\xi}$, then the last term in the equation will be zero or minimized [25,26]. This analysis shows that adding an $\mathrm{rf}$ chirp to the electron beam should improve the emittance exchange. But this analysis ignores CSR which might increase substantially when rf chirp is added to the beam because of the reduction in the bunch length in the dipoles leading to emittance increase.

During the emittance exchange experiment, the beam injected into the EEX beam line has no chirp. We impart a chirp on the beam by operating the 9-cell off crest and measure the bunch length of the electron beam at X24 with a streak camera. For Q3 between 0.35 to $0.55 \mathrm{~A}$, the bunch length reached the resolution limit of the streak camera $(\sim 300 \mathrm{fs})$ as shown in Fig. 12. We then did power measurements with both the bolometer and the pyrodetector for a beam with and without chirp, as shown in Fig. 13. The results indicated a factor of 2 increase in the power detected for a beam with rf chirp. There were 60 bunches and the 5-cell was on in both cases.
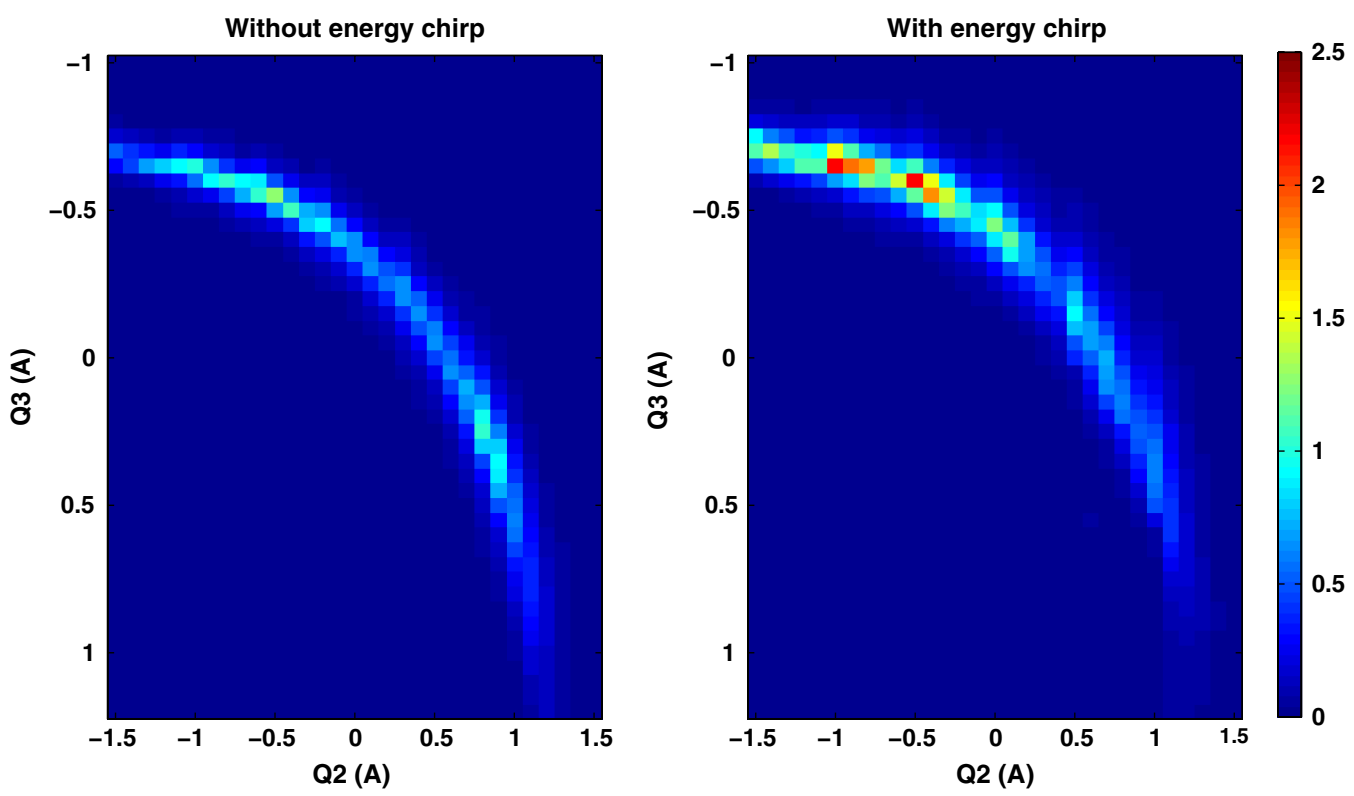

FIG. 13. Pyrodetector measurements of coherent transition radiation at X24 as a function of upstream quadrupoles Q2 and Q3. No energy chirp on the beam (left) and with an energy chirp on the beam (right) at 40 degrees off crest. The intensity obtained when the beam is chirped is a factor of 2 higher compared with the no chirp case at certain quadrupole settings. 


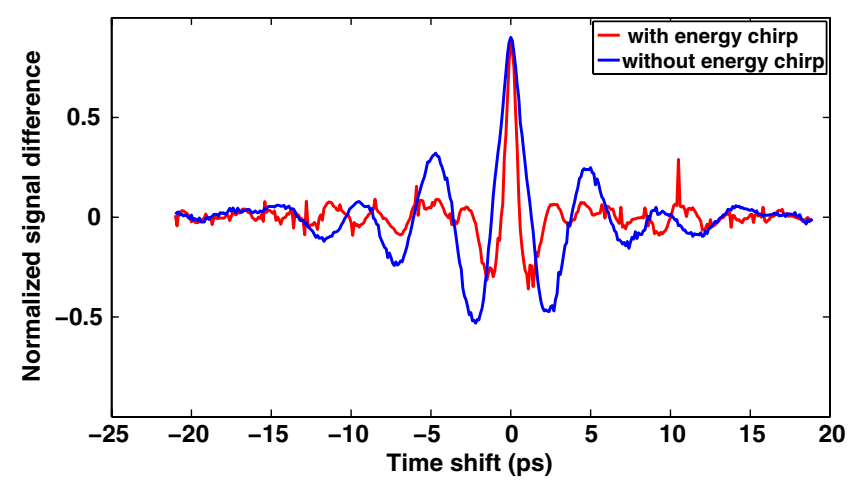

FIG. 14. Autocorrelation measurement with Martin-Puplett interferometer with and without energy chirp.

These results motivated us to operate the EEX beam line with a chirp for the beam and to use the Martin-Puplett interferometer for the bunch-length measurement. The coherent transition radiation (CTR) from X24 was used to measure the autocorrelation of the beam with and without energy chirp. The result is shown in Fig. 14. The bunch with chirp (red) shows a shorter autocorrelation width compared with the bunch with no chirp (blue). Assuming a Gaussian bunch and extracting the rms bunch length from the autocorrelation using the formula $\sigma_{t}=\frac{\mathrm{FWHM}_{\text {center }}}{2.35 \sqrt{2}}$, where FWHM $_{\text {center }}$ corresponds to the full-width half maximum of the center of the autocorrelation peak, yields a bunch length of $0.60 \mathrm{ps}$ (blue) and $0.25 \mathrm{ps}$ (red) [27]. Therefore, adding a chirp decreases the bunch length by a factor of $\sim 2.4$. In other words, the beam with an rf chirp is shorter compared to the one without chirp. This is consistent with the power measurements which also showed a factor of $\sim 2$ increase in the detector CTR power for a beam with chirp and also agrees with the theoretical predictions [28]. Recent emittance measurements shows an improved emittance exchange ratio when operated with an energy-chirped beam [29].

\section{SUMMARY AND CONCLUSIONS}

Coherent synchrotron radiation has been generated, detected, and measured from the emittance exchange beam line at the A0 photoinjector using an energy-chirped beam. The features of CSR such as the angular distribution, the intensity ratio of the horizontal to vertical polarization, and quadratic variation with charge have been presented. A time-resolved measurement of the CSR bulge effect has been measured using the skew-quadrupole at high charge. Finally, by operating the emittance exchange with an energy chirp, a factor of 2 enhancement in peak current has been achieved, demonstrating a possible use of emittance exchange as a bunch compressor. Preliminary results indicate that operating the emittance exchanger with an energy-chirped beam not only shows CSR effects are minimal at lower charges but results in an actual improvement in the emittance exchanger performance. This is consistent with theory and recent simulation [25,30,31]. Further data are being investigated to get an accurate measurement of the emittances after the beam line when operated with a significant energy chirp.

\section{ACKNOWLEDGMENTS}

The authors are indebted to the A0 technical support team. We thank M. Church, V. Shiltsev, H. Edwards, T. Maxwell, P. Piot, and Y.-E. Sun for their interest and

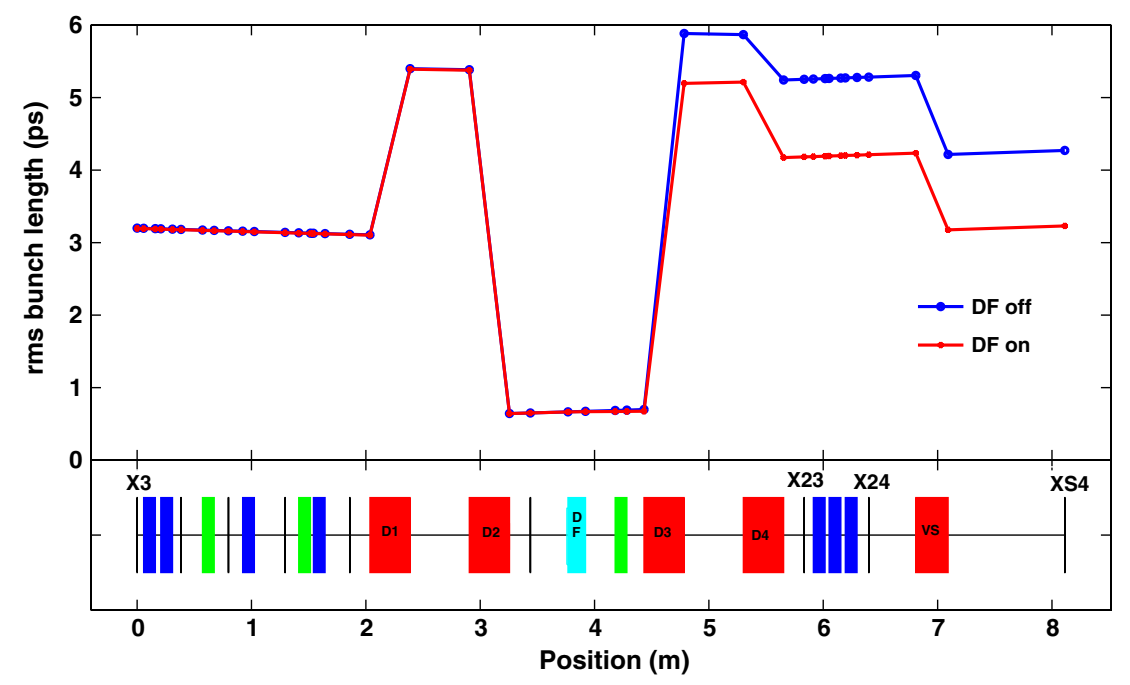

FIG. 15. Evolution of the rms bunch length along the emittance exchange beam line with and without the deflecting cavity powered on. The dipoles (D1, D2, D3, D4) are shown in red. The vertical spectrometer is marked as VS and the deflecting mode cavity is marked as DF. Regular quadrupoles are shown in blue and skew quadrupoles are shown in green. Vertical lines refer to beam imaging stations. 
TABLE II. Beam parameters for the bunch length simulation.

\begin{tabular}{lc}
\hline \hline Parameter & Value \\
\hline Charge & $250 \mathrm{pC}$ \\
Transverse emittance (normalized) & $4 \mu \mathrm{m}$ \\
Longitudinal emittance (normalized) & $18 \mu \mathrm{m}$ \\
Horizontal Twiss parameters: $\alpha_{x}, \beta_{x}$ & $-2.38,25.4 \mathrm{~m}$ \\
Vertical Twiss parameters: $\alpha_{y}, \beta_{y}$ & $-1.85,15 \mathrm{~m}$ \\
Initial bunch length & $3.2 \mathrm{ps}$ \\
\hline \hline
\end{tabular}

encouragement. The work was supported by the Fermi Research Alliance, LLC under the U.S. Department of Energy. One of us (J.T.) would like to thank P. Emma, M. Venturini, J.-L. Vay, and M. Zolotorev for clarifying questions on the 3D effects of CSR.

\section{APPENDIX}

Figure 15 shows the bunch length along the emittance exchange beam line with the rf deflecting mode cavity off and on. The simulation was obtained using BEAMLATTICE for the beam parameters shown in Table II, which models the CSR experiments mentioned in this work. For both cases, the bunch length is minimized at dipole D3, so we expect CSR to be more pronounced at D3 in our experiments.

[1] J. Ruan, A. S. Johnson, A. H. Lumpkin, R. Thurman-Keup, H. Edwards, R. Fliller, T. Koeth, and Y. Sun, Phys. Rev. Lett. 106, 244801 (2011).

[2] M. Cornacchia and P. Emma, Phys. Rev. ST Accel. Beams 5, 084001 (2002).

[3] M. J. Fitch, A. C. Melissinos, P. L. Colestock, J.-P. Carneiro, H. T. Edwards, and W. H. Hartung, Phys. Rev. Lett. 87, 034801 (2001).

[4] P. Piot, Y.-E. Sun, and K.-J. Kim, Phys. Rev. ST Accel. Beams 9, 031001 (2006).

[5] Y.-E. Sun, P. Piot, A. Johnson, A. H. Lumpkin, T. J. Maxwell, J. Ruan, and R. Thurman-Keup, Phys. Rev. Lett. 105, 234801 (2010).

[6] J.-P. Carneiro, N. Barov, H. Edwards, M. Fitch, W. Hartung, K. Floettmann, S. Schreiber, and M. Ferrario, Phys. Rev. ST Accel. Beams 8, 040101 (2005).

[7] J. Schwinger, Phys. Rev. 75, 1912 (1949).

[8] Y. Derbenev, J. Rossbach, E. Saldin, and V. Shiltsev, Report No. TESLA-FEL 95-05, 1995.

[9] G. Stupakov, "Lecture notes on classical mechanics and electromagnetism in accelerator physics,' USPAS School, 2011.
[10] B. Beutner, Report No. DESY-THESIS-2007-040, 2007.

[11] H. H. Braun, R. Corsini, L. Groening, F. Zhou, A. Kabel, T. O. Raubenheimer, R. Li, and T. Limberg, Phys. Rev. ST Accel. Beams 3, 124402 (2000).

[12] K. L. F. Bane, F.-J. Decker, Y. Ding, D. Dowell, P. Emma, J. Frisch, Z. Huang, R. Iverson, C. Limborg-Deprey, H. Loos, H.-D. Nuhn, D. Ratner, G. Stupakov, J. Turner, J. Welch, and J. Wu, Phys. Rev. ST Accel. Beams 12, 030704 (2009).

[13] R. Thurman-Keup, R. Fliller, and G. Kazakevich, in Proceedings of Beam Instrumentation Workshop BIW08 (JaCoW, Lake Tahoe, CA, 2008).

[14] T. Koeth, Ph.D. thesis, Rutgers University, 2009.

[15] H. Wiedemann, Synchrotron Radiation (Springer-Verlag, Berlin, 2003).

[16] C. Behrens, Report No. DESY-THESIS-2010-002, 2008.

[17] G. Carr, M. Martin, W. McKinney, K. Jordan, G. Neil, and G. Williams, Nature (London) 420, 153 (2002).

[18] G. L. Carr, L. DiMauro, W. S. Graves, E. D. Johnson, J. B. Murphy, J. Rudati, B. Sheehy, C. P. Neuman, L. Mihaly, and D. Talbayev, in Proceedings of the Particle Accelerator Conference, Chicago, IL, 2001 (IEEE, New York, 2001).

[19] An in-house software based on TRACE3D linear matrix model for beam transport with a GUI interface. This code was written in MATLAB by R. Thurman-Keup.

[20] M. Borland, Advanced Photon Source LS-287, 2000.

[21] L. Fröhlich, TESLA FEL Report No. 2, 2005.

[22] R. Lai and A. Sievers, Nucl. Instrum. Methods Phys. Res., Sect. A 397, 221 (1997).

[23] K. Bertsche, P. Emma, and O. Shevchenko, Report No. SLAC-PUB-13614.

[24] Y.-E. Sun, P. Piot, A. Johnson, A. H. Lumpkin, T. J. Maxwell, J. Ruan, and R. Thurman-Keup, Phys. Rev. Lett. 105, 234801 (2010).

[25] P. Emma, Z. Huang, K.-J. Kim, and P. Piot, Phys. Rev. ST Accel. Beams 9, 100702 (2006).

[26] B. Jiang, J. G. Power, R. Lindberg, W. Liu, and W. Gai, Phys. Rev. Lett. 106, 114801 (2011).

[27] R. Trebino, Frequency-Resolved Optical Gating: The Measurement of Ultrashort Laser Pulses (Springer, New York, 2000), Vol. 1.

[28] P. Piot, L. Carr, W. S. Graves, and H. Loos, Phys. Rev. ST Accel. Beams 6, 033503 (2003).

[29] J. C. T. Thangaraj, J. Ruan, A.S. Johnson, R. ThurmanKeup, A. H. Lumpkin, J. Santucci, Y.-E. Sun, and H. Edwards, in Proceedings of the International Particle Accelerator Conference, Kyoto, Japan (ICR, Kyoto, 2010).

[30] B. E. Carlsten, K. A. Bishofberger, S. J. Russell, and N. A. Yampolsky, Phys. Rev. ST Accel. Beams 14, 084403 (2011).

[31] A. Zholents and M. Zolotorev, Advanced Photon Source Report No. ANL/APS/LS-327, 2011. 